\title{
Pharmacological Modulation of Behavioral and Neuronal Correlates of Repetition Priming
}

\author{
Christiane M. Thiel, ${ }^{1}$ Richard N. A. Henson, ${ }^{1,2}$ John S. Morris, ${ }^{1}$ Karl J. Friston, ${ }^{1}$ and Raymond J. Dolan ${ }^{1,3}$ \\ ${ }^{1}$ Wellcome Department of Cognitive Neurology, Institute of Neurology, London, WC1 3BG, United Kingdom, 2Institute of \\ Cognitive Neuroscience, University College London, London WC1 3BG, United Kingdom, and ${ }^{3 R o y a l ~ F r e e ~ H o s p i t a l ~}$ \\ School of Medicine, London NW3 2PF, United Kingdom
}

In this experiment we address the pharmacological modulation of repetition priming, a basic form of learning, using eventrelated functional magnetic resonance imaging. We measured brain activity in a word-stem completion paradigm in which, before study, volunteers were given either placebo, lorazepam (2 mg orally), or scopolamine (0.4 mg, i.v.). Relative to placebo, both drugs attenuated the behavioral expression of priming. Repetition was associated with a decreased neuronal response in left extrastriate, left middle frontal, and left inferior frontal cortices in the placebo group. Both drugs abolished these "repetition suppression" effects. By showing a concurrence of behavioral and neuronal modulations, the results suggest that GABAergic and cholinergic systems influence the neuronal plasticity necessary for repetition priming.

Key words: fMRl; priming; scopolamine; lorazepam; psychopharmacology; neuroimaging; word-stem completion; repetition suppression
Previous exposure to a stimulus facilitates or biases its subsequent processing. This behavioral phenomenon is known as repetition priming and is likely to constitute a basic learning mechanism (Wiggs and Martin, 1998; Schacter and Buckner, 1998). One possible neuronal signature for this form of learning is "response suppression," a decrement in the response to repeated stimuli in neurons that fire to initial presentations (Desimone, 1996). In monkeys, for example, a subpopulation of cells in inferior temporal cortex shows reduced neuronal firing when novel stimuli are repeated ( $\mathrm{Li}$ et al., 1993). In humans, analogous decreases in hemodynamic response after repetition in brain areas such as extrastriate and frontal cortices have been demonstrated with neuroimaging methods (Squire et al., 1992; Buckner et al., 1998, 2000; Wagner et al., 2000).

Cholinergic mechanisms exert a regulatory function with respect to the expression of plasticity (Brocher et al., 1992; Weinberger, 1998; Rasmusson, 2000) and learning (Blokland, 1996). Although cholinergic modulation of learning, and corresponding neuronal activity, has been shown in many explicit learning paradigms (Caine et al., 1981; Frith et al., 1984; Nissen et al., 1987; Rusted and Warburton, 1988; Curran et al., 1991a; Furey et al., 2000), its effects on behavioral measures in implicit paradigms is more controversial. Psychopharmacological and electrophysiological evidence suggests an absence of cholinergic modulation on repetition priming, because several studies found no behavioral (Danion et al., 1990; Knopman, 1991; Schifano and Curran, 1994)

Received April 26, 2001; revised June 19, 2001; accepted June 19, 2001.

This work was supported by program grants from the Wellcome Trust to R.J.D. and K.J.F., a Wellcome Trust Fellowship to R.N.A.H., and a research grant from the German Research Foundation (Deutsche Forschungsgemeinschaft) to C.M.T. We thank the radiographers at the Functional Imaging Lab for help with fMRI scanning and medical colleagues for help with injections. Stimuli for the word-stem completion task were kindly provided by R. D. Badgaiyan. Thanks to Richard Frackowiak for his helpful comments on an earlier version of this manuscript.

Correspondence should be addressed to Christiane Thiel, Wellcome Department of Cognitive Neurology, Institute of Neurology, 12 Queen Square, London, WC1 3BG, UK. E-mail: cthiel@fil.ion.ucl.ac.uk.

Copyright (ㄷ) 2001 Society for Neuroscience $\quad 0270-6474 / 01 / 216846-07 \$ 15.00 / 0$ or neuronal (Miller and Desimone, 1993) modulation of repetition effects after administration of the cholinergic antagonist scopolamine. By contrast, manipulations of GABAergic neurotransmitter systems with the benzodiazepine lorazepam impairs behavioral indices of repetition priming in humans (Knopman, 1991; Buffett-Jerrott et al., 1998; Vidailhet et al., 1999).

In this study, we address the pharmacological modulation of repetition priming at both the behavioral and neuronal levels. We combined event-related functional magnetic resonance imaging (fMRI) with drug challenge in a word-stem completion paradigm in which the behavioral index of priming was a bias in the completions of word-stems in favor of previously presented words. Results from previous neuroimaging studies, using wordstem completion, enabled specific predictions of repetition suppression in left extrastriate, left inferior, and middle frontal cortex (Buckner et al., 2000). Thus, the experimental question of interest was whether lorazepam and scopolamine would modulate repetition suppression in these regions.

\section{MATERIALS AND METHODS}

Subjects. Forty-seven right-handed native English-speaking participants (29 male, 18 female; age range, 18-37 years) with no history of medical or psychiatric disease gave informed consent to participate in the study. We excluded 10 participants from analysis. Exclusion criteria were technical failure $(n=2)$, excessive head movement $(n=2)$, or $<60 \%$ of word stems completed $(n=6)$. The effective group size was $n=13$ for placebo ( 8 male, 5 female; mean age, $23.92 \pm 1.22$ ), $n=12$ for lorazepam ( 9 male, 3 female; mean age, $23.00 \pm 1.37$ ), and $n=12$ for scopolamine ( 6 male, 6 female; mean age, $24.08 \pm 1.34$ ).

Drugs. A double-blind, double-dummy, time-elapsed drug administration technique was used, so that each subject received a tablet orally and an injection intravenously 120 and $80 \mathrm{~min}$, respectively, before the start of the study phase, i.e., (1) placebo orally plus saline intravenously, (2) 2 $\mathrm{mg}$ of lorazepam orally plus saline intravenously, or (3) placebo orally plus $0.4 \mathrm{mg}$ of scopolamine intravenously. Drug doses and administration schedule were chosen according to the literature to induce behavioral impairments. Doses between 2 and $3 \mathrm{mg}$ of oral lorazepam have been shown to reliably impair repetition priming, whereas lower doses are less effective (Knopman, 1991; Legrand et al., 1995; Buffett-Jerrott et al., 1998). Maximal effects occur at $2 \mathrm{hr}$ after ingestion, which also corre- 
sponds to peak plasma levels (Legrand et al., 1995). Scopolamine was administered intravenously because oral scopolamine has variable absorption, poor bioavailability, and is behaviorally less effective (Nuotto, 1983; Putcha et al., 1989). The dose of scopolamine was chosen because intravenous injections in this dose range have been reported to affect a variety of cognitive functions (Sunderland et al., 1987; Martinez et al., 1997; Vitiello et al., 1997). Scopolamine was administered $80 \mathrm{~min}$ before scanning, because previous studies have shown that cognitive effects of intravenous scopolamine peak from 90 to $150 \mathrm{~min}$ after administration (Safer and Allen, 1971; Sannita et al., 1987; Curran et al., 1991b; Ebert et al., 1998).

Experimental paradigm. Stimuli were selected from a pool of 200 target words so that the initial three letters were unique and could be completed by at least six English words (Badgaiyan et al., 1999). Stimuli were randomly divided into two sets of 100 words, A and B. Set A was used during scanning to index priming-related brain activity, whereas set $\mathrm{B}$ was used after scanning to obtain a behavioral measure of priming. Both sets were divided again into two subsets. During the prescanning study phase, all words of one or the other of the subsets of list A were displayed every $5 \mathrm{sec}$ for $5 \mathrm{sec}$ on a Macintosh computer, and subjects were asked to read the words aloud and rate them by pleasantness. At test, participants were presented with a completion task for the 50 stems of the words presented in the study phase ("old word-stems") randomly intermixed with stems of nonpresented words ("new word-stems"). The assignment of subsets to old or new items was counterbalanced across subjects, so that completions for the new stems in one subject acted as the baseline for another in which that subset comprised the old items. Stimuli [including 50 "null events" (Josephs and Henson, 1999)] were displayed every $4 \mathrm{sec}$ for $1 \mathrm{sec}$ against a baseline of fixation crosses on a Macintosh computer, projected onto a screen $\sim 300 \mathrm{~mm}$ above the subject in the magnetic resonance imaging (MRI) scanner. Participants were asked to complete the stems covertly with the first word that came to mind and make a right index finger key press for every completion.

For postscanning word-stem completion, participants were presented with words of one or other of the subsets of list B and asked to read the words aloud. One half of those words was rated by pleasantness, and the other half was counted for the presence of letters with enclosed spaces (e.g., an "a" contains an enclosed space, but an "r" does not). The aim of processing half the words in the study phase "deeply" (rating by "liking") opposed to "shallowly" (counting enclosed spaces) was to determine the contribution of explicit memory processes to task performance (Graf and Mandler, 1984). During the test phase, completion of word stems was overt. The comparison of completions of old word-stems with previously presented words and new word-stems with words from the nonpresented subset provided a behavioral index of repetition priming. Behavioral data were analyzed with a three $\times$ two-way ANOVA for repeated measures with drug as a between-subject factor and priming (new vs old wordstems) as a within-subject factor. Significant effects were followed by post hoc Tukey tests comparing priming between and within groups.

Imaging and image processing. A VISION MRI system (Siemens, Erlangen, Germany) operating at $2 \mathrm{~T}$ was used to obtain $\mathrm{T} 2 *$-weighted echoplanar (EPI) images $(64 \times 64,3 \times 3 \mathrm{~mm}$ pixels $)$ with blood oxygenation level-dependent (BOLD) contrast. Two hundred and fifty volumes of 302 -mm-thick axial slices were acquired sequentially every $3.5 \mathrm{~mm}$ (repetition time, $2.5 \mathrm{sec}$; echo time, $40 \mathrm{msec}$ ). The first five volumes were discarded to allow for T1 equilibration effects. Images were realigned to correct for interscan movement, synchronized to the middle slice to correct for differences in slice acquisition time, and normalized to a standard EPI template volume. The data were then smoothed with a Gaussian kernel of $8 \mathrm{~mm}$ full-width half-maximum to accommodate intersubject anatomical variability.

Statistical analysis of images. Data were analyzed with statistical parametric mapping software (SPM99; Wellcome Department of Cognitive Neurology, London, UK; Friston et al., 1995) using a random effects analysis. Data were globally scaled to 100 across scans and highpassfiltered at $1 / 60 \mathrm{~Hz}$. The hemodynamic response to stimulus onset for each event type was modeled by a canonical synthetic hemodynamic response function (HRF) and its first-order temporal derivative. Three event types were modeled (new and old words as effects of interest and missed responses as effect of no interest). The six head movement parameters were included as confounds. Linear contrasts of parameter estimates for the canonical HRF for each subject were taken to a second level analysis to generate statistical parametric maps (SPMs) of the $t$-statistic. An SPM (thresholded at $p<0.001$, uncorrected) of voxels showing significant responses during task performance (i.e., main effect) was created and

\section{Study Phase}
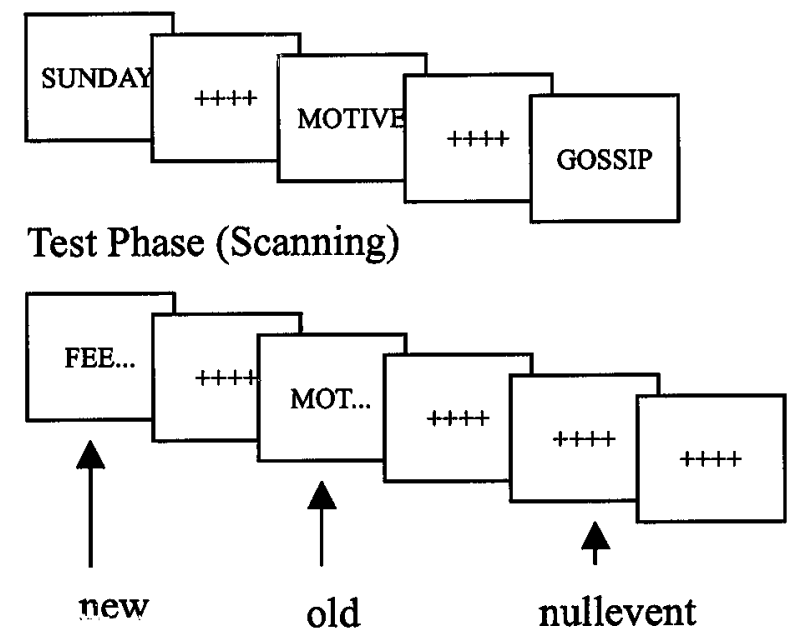

Figure 1. Experimental paradigm. Illustration of stimuli presented during study and test phases. Test stimuli were either word-stems that could be completed with words presented in the study phase (old) or stems that could not (new). Stimuli were randomly displayed every $4 \mathrm{sec}$ for $1 \mathrm{sec}$ against a baseline of fixation crosses.

used as a mask to identify brain regions showing repetition suppression and group by repetition interactions. Additionally, percentage of signal change for the effect maxima in the three regions of interest (left extrastriate, left middle frontal, and left inferior frontal cortices), showing a drug by repetition interaction, were plotted for each group. These data were analyzed in the same way as the behavioral data, i.e., with a three $\times$ two-way ANOVA for repeated measures followed by post hoc Tukey's tests.

\section{RESULTS}

Subjects were randomly allocated to three groups and given either placebo, lorazepam (2 mg oral), or scopolamine ( $0.4 \mathrm{mg}$, i.v.). In a study phase, conducted outside the scanner, and after drug or placebo administration, subjects were presented visually with a list of words and asked to read them aloud and rate them for pleasantness. Scanning took place during a subsequent test phase, during which subjects performed a completion task in relation to three-letter stems of words presented at study (old word-stems) randomly intermixed with stems of nonpresented words (new word-stems) (Fig. 1). To minimize response-related head movement, participants were asked to complete stems covertly with the first word that came to mind and make a right index finger key press after every completion. Covert word-stem completion has been found to yield similar repetition priming effects to overt word-stem completion (Buckner et al., 2000). Immediately after scanning, the task was repeated with a new set of stimuli and overt stem completion to obtain a behavioral measure of priming.

Analysis of behavioral data with a three $\times$ two-way ANOVA for repeated measures yielded a drug by repetition interaction $\left(F_{(2,34)}=6.32 ; p=0.005\right)$ (Fig. 2). Relative to placebo, priming was significantly attenuated, although not abolished, in both drug groups ( post hoc Tukey tests old word-stems in drug groups vs old word-stems in placebo group, $p<0.05$; post hoc Tukey tests new word-stems vs old word-stems in the lorazepam and scopolamine group, $p<0.05)$. This indicates that repetition priming in wordstem completion paradigms is sensitive to both cholinergic and GABAergic drug manipulation.

To determine whether sedation contributed to these effects, we (1) compared the total number of completed word-stems (old and 


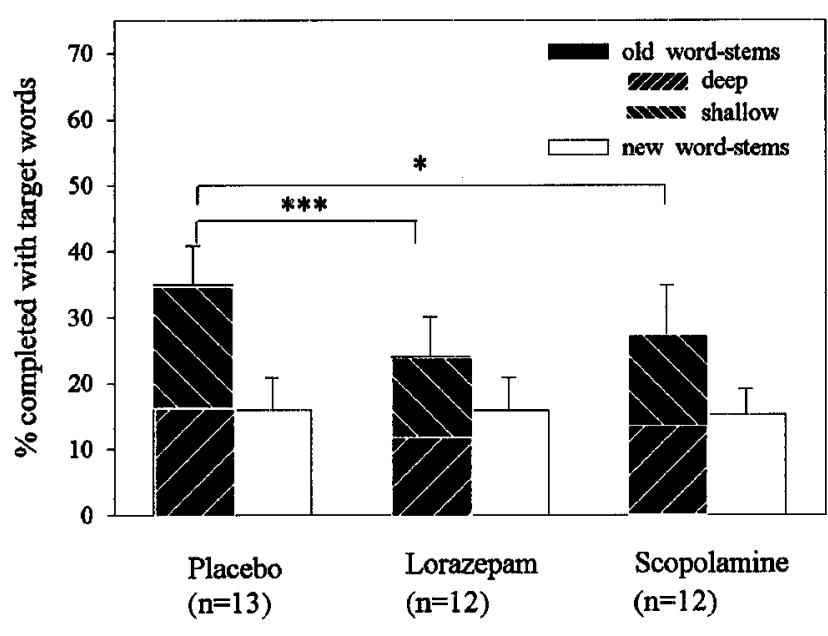

Figure 2. Behavioral performance in word-stem completion paradigm. Mean and SEM of word-stems completed with target words from the previously presented list (old; subdivided into deep and shallow) and words from previously nonpresented list (new $=$ chance completion). Note that, for purposes of illustration in deep and shallow conditions, percentages refer to percentage of stems completed from the whole previously presented list (irrespective of how words were encoded-i.e., the maximum value in both conditions would be 50\%). Data were analyzed with ANOVAs for repeated measures, with drugs as a betweensubject factor and priming (new vs old stems) as a within-subject factor. Significant effects were followed by post hoc Tukey's tests comparing placebo and drug groups $\left({ }^{*} p<0.05\right.$; *** $\left.p<0.001\right)$.

new) in placebo and drug groups and (2) correlated subjective sedation with the behavioral index of repetition priming. Subjective sedation was assessed with visual analog scales (Bond and Lader, 1974). There was neither a group difference in total number of completed word-stems $\left(F_{(2,34)}=1.101 ; p=0.344\right)$ nor a correlation between measures of sedation and priming $(r=$ $-0.111 ; p=0.514)$. A second concern was that attenuation of repetition priming might reflect the action of lorazepam and scopolamine on explicit memory. As described (see Materials and Methods), we included a deep and shallow encoding condition in the postscanning word-stem completion (Graf and Mandler, 1984). The absence of a difference in priming after deep or shallow encoding $\left(F_{(1,34)}=0.354 ; p=0.556\right)$ suggests that priming effects were mediated primarily by implicit memory processes. Consequently, it is unlikely that drug effects reflect an impairment of explicit memory processes.

To illustrate the overall task effect in all three groups brain regions showing activity during word-stem completion compared with fixation are shown in Figure 3. A network of regions were activated in all groups, including bilateral extrastriate regions, left frontal regions along the inferior frontal gyrus, the anterior cingulate, and the bilateral cerebellar cortex, confirming results from previous fMRI studies (Buckner et al. 2000). Furthermore, these findings are in line with our behavioral data, showing that task performance (i.e., the number of completed word-stems) was not different between groups.

Because both drugs impaired the behavioral expression of repetition priming, our main question was whether these effects are expressed in a modulation of the neuronal index of repetition suppression. This question was addressed in two planned comparisons. First, we identified brain regions showing significant repetition suppression under placebo. Second, we compared the magnitude of these reductions by contrasting the placebo and drug groups (averaging across scopolamine and lorazepam), i.e., we tested for a group (placebo and drug) by repetition interaction. In the placebo group (Fig. $4 a$ ), repetition-related decreases were evident in several brain areas, including left extrastriate cortex, left inferior frontal cortex, and left middle frontal cortex, regions previously shown to manifest repetition suppression effects (Buckner et al., 2000). A comparison of repetition-related effects in placebo and the combined drug groups revealed a significant interaction in these same regions, including left extrastriate, left middle frontal, and to a lesser extent, left inferior frontal cortex (Fig. 4b). This drug-by-repetition interaction reflected an absence of repetition suppression after lorazepam and scopolamine. In other words, repetition suppression was impaired in the presence of the two pharmacological agents. Consequently, GABAergic and cholinergic influences on repetition priming in word-stem completion paradigms are expressed as an attenuation of repetition suppression in the same brain areas associated with repetition effects in the placebo group and in previous studies using this paradigm (Buckner et al., 2000).

One possible issue of concern in a drug study is that of sedation. To determine whether sedation contributed to these neuronal effects, we correlated subjective sedation with the neuronal index of repetition priming. There was no correlation between measures of sedation and repetition suppression in left extrastriate $(r=-0.20 ; p=0.235)$, left middle frontal $(r=-0.036 ; p=$ $0.831)$, and left inferior frontal cortex $(r=-0.121 ; p=0.477)$. An additional concern is the possibility of drug effects on global perfusion, consequently to investigate whether the drugs might have interfered with global cerebral blood flow ( $\mathrm{gCBF})$, we compared the estimated global BOLD signal across the brain between the drug groups. No differences in this global estimate were found $\left(\right.$ ANOVA, $\left.F_{(2,34)}=0.406 ; p=0.669\right)$.

A further exploration of the data were performed to evaluate (1) the contribution of lorazepam and scopolamine to the drug by repetition interaction and (2) the mechanisms underlying the absence of repetition suppression. Theoretically an absence of repetition suppression might be driven by an increased responses to old word-stems in the drug groups relative to responses to old word-stems in the placebo group or decreased responses to new word-stems in the drug groups relative to responses to new wordstems in the placebo group. In Figure $5 A-C$ we plot the percentage of signal change for the effect maxima separately for the three drug groups. A statistical analysis identical to that for behavioral data (i.e., three $\times$ two-way ANOVA for repeated measures followed by post hoc Tukey tests) showed that both drugs interfered with repetition suppression in left extrastriate (ANOVA drug by repetition interaction, $\left.F_{(2,34)}=7.595, p=0.002\right)$, left middle frontal (ANOVA drug by repetition interaction, $F_{(2,34)}=7.772$, $p=0.002$ ), and left inferior frontal cortex (ANOVA drug by repetition interaction, $\left.F_{(2,34)}=6.109 ; p=0.005\right)$.

Differences were apparent in the nature of the interference with repetition suppression, i.e., different modulation of neuronal responses to old or new items by lorazepam and scopolamine when compared with placebo. For lorazepam, the interference with repetition suppression was driven primarily by reduced activation to new word-stems in left extrastriate and frontal cortex (Tukey tests against placebo, $p<0.05$ ). Scopolamine, on the other hand, interfered with repetition suppression in left extrastriate cortex by significantly increasing responses to old wordstems with respect to placebo (Tukey tests against placebo, $p<$ 0.05 ), while leaving responses to new word-stems intact. Intact responses to new word-stems were also seen in inferior frontal cortex. To determine whether these patterns of impaired repeti- 

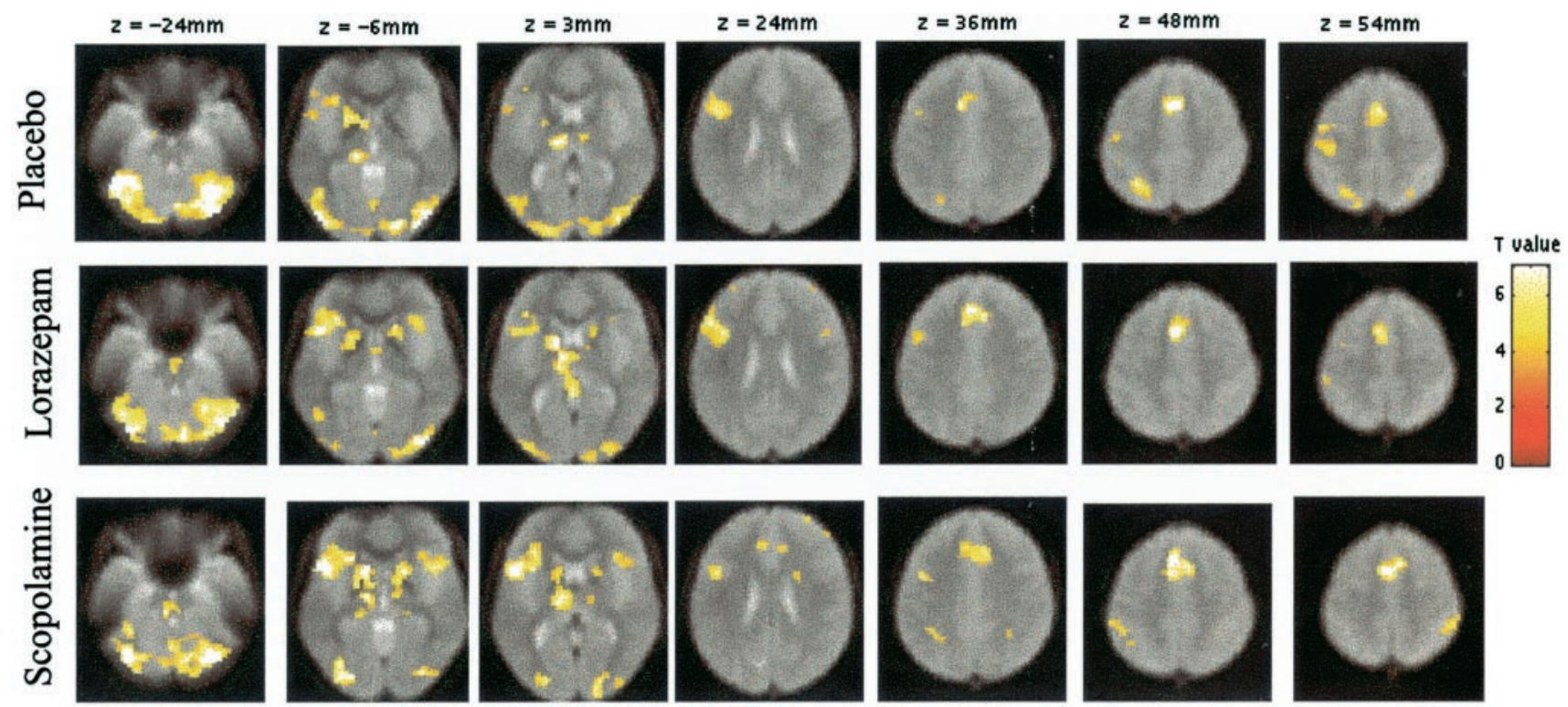

Figure 3. Regions showing activations during word-stem completion (new and old word-stems) against fixation, as identified by random effects analysis in the placebo, lorazepam, and scopolamine groups, respectively. Activations are rendered on transverse mean normalized EPI images (EPI images averaged over several volunteers; threshold $=p<0.001$ ). EPI images are used for anatomical descriptions, because they show the same distortions as activation data. A pathway of regions is activated, including bilateral extrastriate regions, left frontal regions along the inferior frontal gyrus, the anterior cingulate, and bilateral cerebellar cortex. The left lateral parietal cortex and precentral gyrus were only significantly activated in the placebo group.
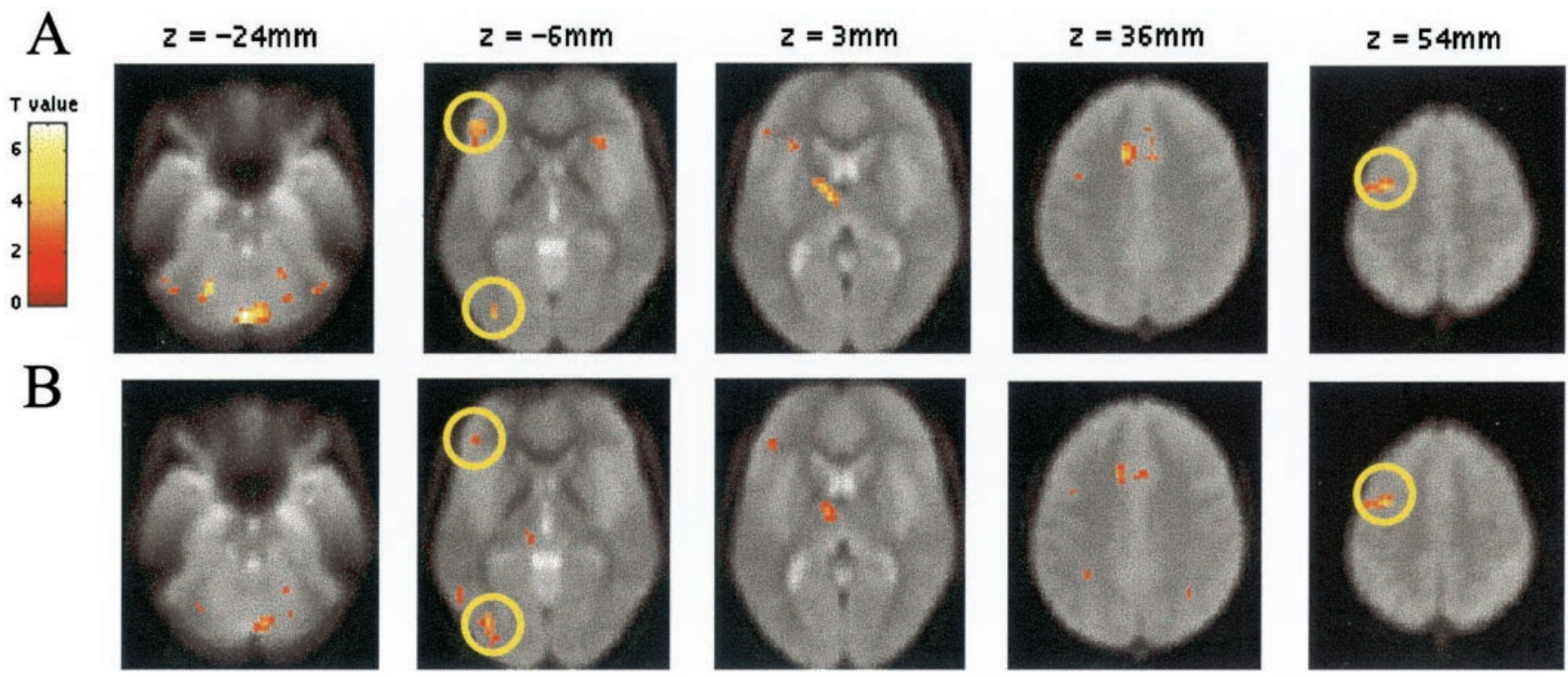

Figure 4. Regions showing repetition suppression, as identified by random effects analysis. Activations (thresholded at $p<0.01$ for purposes of illustration) are rendered on transverse mean normalized EPI images. $A$, Regions showing repetition-related activation reductions [(placebo new-old), i.e., increased activity for new compared with old word-stems] in the placebo group. Regions highlighted by circles are: left inferior frontal cortex ( -48 , $39,-6 ; Z=3.22)$, left extrastriate cortex $(-36,-81,-6 ; Z=3.14)$, and left middle frontal cortex $(-30,0,57 ; Z=3.67)$. Other areas activated at $p<$ 0.001 include: cerebellum $(0,-78,-24, Z=4.67$ and $-21,-60,-24, Z=3.98)$, left thalamus $(-9,-9,3 ; Z=3.63)$, left cingulate $(-12,18,36 ; Z=$ $3.64)$, right caudate $(18,6,18 ; Z=3.56)$, right extrastriate $(39,-66,-30 ; Z=3.39)$, and right inferior frontal cortex $(30,27,-9 ; Z=3.26)$. $B$, Regions showing a repetition by drug interaction [i.e., (placebo new-old) - (drug new-old)]. Regions highlighted are: left inferior frontal cortex $(-45,39,-9$; $Z=3.06)$, left extrastriate cortex $(-36,-75,-6 ; Z=3.73)$, and left middle frontal cortex $(-39,-3,54 ; Z=3.67)$. Further areas activated at $p<0.001$ include: right cerebellum $(9,-75,-24 ; Z=3.7)$, left cingulate $(-12,15,36 ; Z=3.51)$, left thalamus $(-9,9,3 ; Z=3.24)$, and left inferior parietal cortex $(-30,-51,48 ; Z=3.08)$. In all these regions both drugs lead to a significant decrease in repetition suppression compared with placebo. When we used regions showing repetition suppression in the placebo group as a mask within which to identify a group $\times$ repetition interaction several areas survived a small volume correction based on this search volume. These included: left extrastriate cortex $(p=0.015$, corrected $)$, left middle frontal cortex $(p=$ 0.021 , corrected), cingulate ( $p=0.023$, corrected $)$, and cerebellum $(p=0.012$, corrected $)$. 

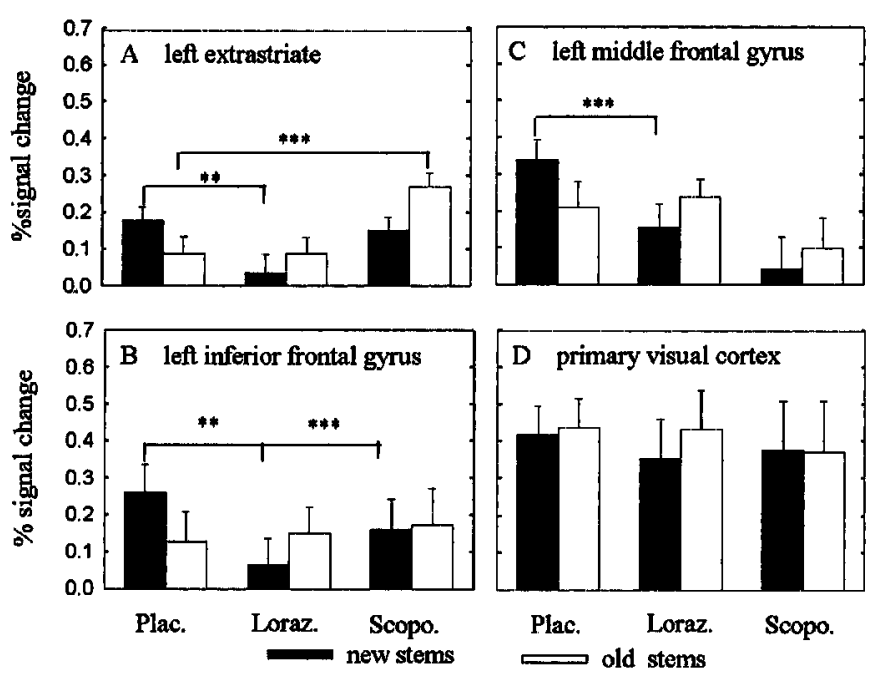

Figure 5. Plots of percentage of signal change. Mean and SEM as a function of group and repetition (new stems, black symbols; old stems, white symbols). The plots in $A-C$ derive from the maximum voxel in each region identified by random effects analysis. $D$ shows a voxel in primary visual cortex. The placebo group shows repetition suppression in left extrastriate, left inferior frontal, and left middle frontal cortices. There is an absence of repetition-related reductions in all three brain areas after lorazepam and scopolamine. Compared with placebo, lorazepam reduced activations to new words in all three brain regions. In contrast, scopolamine showed increased activations to old words compared with placebo in extrastriate cortex. Note that the voxel in primary visual cortex shows neither repetition suppression nor an effect of drug. $* * p<0.05 ; * * * p<$ 0.001 ; ANOVAs followed by post hoc Tukey's tests comparing activations to new and old words between placebo and drug groups.

tion priming were significantly different between lorazepam and scopolamine, we directly compared the two drug groups using a two $\times$ two-way ANOVA for repeated measures. We found a significant drug effect in extrastriate cortex showing greater activation under scopolamine compared with lorazepam (ANOVA main effect drug, $\left.F_{(1,22)}=8.797, p=0.007\right)$. No significant drug effects or drug by repetition interactions were found for other brain regions (ANOVAs, $p>0.05$ ).

To determine whether the drugs nonspecifically interfered with brain activity during task performance, results from a voxel in primary visual cortex $(12,-90,-6)$, which showed stimulusdriven responses during word-stem completion, were plotted (Fig. $5 D$ ). If the action of lorazepam or scopolamine was nonspecific, drug-related modulations would be expected in this primary visual brain area. No group (ANOVA, $F_{(2,34)}=0.069, p=0.933$ ), or group by repetition interaction (ANOVA, $F_{(2,34)}=0.481, p=$ 0.622 ) was evident in primary visual cortex, indicating that the drugs did not modulate activity in primary visual areas.

\section{DISCUSSION}

Our behavioral findings indicate that both lorazepam and scopolamine influence the behavioral expression of word-stem completion priming, whereas neuroimaging data provide evidence that this same modulation impairs repetition suppression. The findings thus provide evidence for specific neuronal events associated with drug-attenuated repetition priming during word-stem completion.

An important theoretical consideration is the possibility that drugs, such as scopolamine, might affect $\mathrm{gCBF}$ and/or regional cerebral blood flow (rCBF). Tsukada et al. (1997) have shown in monkeys that $\mathrm{rCBF}$ to somatosensory stimulation is abolished by scopolamine. The doses shown to yield this effect were however 10 times higher than the dose used here, and of critical relevance is the fact that no effects on $\mathrm{rCBF}$ were found at a lower dose. To account for possible differences in gCBF between the groups in our data, global scaling was used in the data analysis. Furthermore, we found no evidence for a difference when we compared the estimated global BOLD signal between the drug groups, which makes significant changes in gCBF unlikely. Another concern might be fluctuation in drug effects over the time course of the experiment. In relation to the half-life of lorazepam $(8-25 \mathrm{hr})$ (Greenblatt, 1981), only minor fluctuations can be expected during the course of the experiment. The half-life of scopolamine (220 $\mathrm{min})$ is a potentially greater concern, and a decline in drug levels might occur between scanning and behavioral testing. However, because we found robust behavioral effects after scanning, we can, at a minimum, be confident that the drug was still centrally active. Additionally a study investigating the cerebral distribution kinetics of radioactively labeled scopolamine showed only minor changes in cortical scopolamine concentration between 60 and 120 min after injection, with still-increasing activity at the latter time point (Frey et al., 1992). On this basis we can infer that drug fluctuations between imaging and postscanning behavioral measures are negligible.

The attenuation of behavioral indices of repetition priming with lorazepam is consistent with previous psychopharmacological studies (Buffett-Jerrott et al., 1998; Vidailhet et al., 1999). In contrast to most previous studies, however (Danion et al., 1990; Knopman, 1991; Schifano and Curran, 1994), our results with scopolamine also demonstrate a cholinergic modulation of repetition priming. We suggest that these differences could be attributable to a lower active dose used in these previous studies (subcutaneous or intramuscular injections are centrally less effective) so that priming was not significantly impaired. Indeed the study by Schifano and Curran (1994), which used two doses of scopolamine, found a tendency toward attenuated repetition priming at higher doses of the drug.

A lack of repetition suppression after lorazepam and scopolamine was found in those brain areas associated with repetition effects in the placebo group, suggesting that GABAergic and cholinergic systems influence repetition priming through modulation of neuronal plasticity in these brain areas. We do not have an explanation why the remaining priming effect in the drug groups co-occurred with a lack of repetition suppression, it might however simply reflect a lack of power to pick up repetition suppression with low levels of priming.

The disruption of repetition suppression with scopolamine might seem inconsistent with electrophysiological data of Miller and Desimone (1993) where no effect on repetition suppression was evident. Important methodological differences in these studies are likely to account for these apparent discrepancies. First, the recording sites in the former study were more anterior than activations we report in extrastriate cortex. Second, the time interval between first and second presentation of the stimuli was different. In Miller and Desimone (1993), the first and second presentation of the stimuli was less than a minute apart, whereas in our study the first presentation of words in the study phase and the presentation of old word-stems during scanning were separated by a much longer interval ( $\sim 40 \mathrm{~min})$. Because repetition suppression can be sensitive to lag (Henson et al., 2000), it may be that weaker repetition suppression with longer lags is more sensitive to drug influences. Indeed, Nissen et al. (1987) found that word fragment completion was impaired by scopolamine when 
there was a 60 min delay between study and test phase but not when the delay was 5 min (although the authors attributed these lag effects to an influence of explicit memory).

The extrastriate region, and its extension into inferior temporal cortex, is an area that shows robust repetition suppression in word-stem completion paradigms (Schacter and Buckner, 1998). It has been argued that repetition suppression in this area is independent of stimulus modality, because it is seen with visual and auditory word-stem completion tasks and thus reflects processes beyond mere perceptual facilitation (Badgaiyan et al., 1999; Buckner et al., 2000). Lorazepam is known to impair perceptual processes (Wagemans et al., 1998), and its behavioral effects on priming have often been argued to be purely of perceptual nature (Giersch et al., 1995). The present lack of repetition suppression in a putative "amodal" left extrastriate region suggests impairments beyond a pure perceptual level of processing (e.g., at a lexical level; Richardson-Klavehn and Gardiner, 1998). The action of lorazepam on such an amodal level of processing is consistent with previous data showing that lorazepam impairs both visual and auditory word-stem completion (Vidailhet et al., 1999). The proposal that behavioral deficits produced by both drugs reflect influences beyond the perceptual level is further supported by their effects in frontal areas.

Previous neuroimaging studies have also reported repetition suppression in left inferior frontal cortex with repeated semantic word classification, repetitive word generation, and word-stem completion tasks (Demb et al., 1995; Thompson-Schill et al., 1999; Buckner et al., 2000). This phenomenon could reflect increased efficiency of access, retrieval, or selection of semantic information. An attenuation of repetition suppression in frontal areas with both drugs suggests that subjects treated with lorazepam and scopolamine might not benefit from previous semantic processing.

Even if differential effects of lorazepam and scopolamine are weak when compared with each other, trends in our data suggest a need to investigate possible differential modulation of neuronal responses by GABAergic and cholinergic systems. Although both drugs impaired repetition suppression in critical brain areas, there were subtle differences in the drugs' action when compared with placebo. Lorazepam reduced activation to new word-stems in all three brain areas, a result that suggests that lorazepam attenuated responses to words in the study phase, which were all novel. Although the task during study phase was different from test, such a conjecture would be consistent with psychopharmacological evidence, that lorazepam impairs word-stem completion when it is given before, but not after, a study phase (Vidailhet et al., 1994). In other words, lorazepam may affect initial processing of stimuli (e.g., at a lexical level; Richardson-Klavehn and Gardiner, 1998) and the associated neuronal responses in left extrastriate and frontal regions. However, whether insufficient activation with initial stimulus presentation after lorazepam also reduces the magnitude of neuronal activations during subsequent processing of these stimuli (e.g., activations to old word-stems) needs further investigation.

Scopolamine, on the other hand increased activations to old word-stems, at least in left extrastriate cortex. Response enhancements with repeated stimulus presentations have previously been found in right fusiform cortex for repetition of unfamiliar or degraded faces and might reflect additional processing (George et al., 1999; Henson et al., 2000). Behaviorally, repetition of unfamiliar faces induced less repetition priming, as measured by reaction times, than repetition of familiar faces (Henson et al.,
2000). Repetition enhancements to old words in a word-stem completion paradigm have been observed in right extrastriate regions of Alzheimer's patients, who show behaviorally similar priming impairments as our scopolamine subjects (Backman et al., 2000). Thus, repetition-related response enhancements instead of decreases in extrastriate areas are also observed in other conditions that show reduced repetition priming.

\section{Conclusion}

We demonstrate that GABAergic and cholinergic systems influence the behavioral expression of word-stem completion priming. This behavioral change was associated with an impairment of repetition suppression in left extrastriate, left middle frontal, and left inferior frontal cortices, thus pointing to a locus of the drugs' cerebral action in word-stem completion paradigms.

At a broader level the present study is a first step to investigate the pharmacological basis of repetition suppression and behavioral priming in humans. Although further studies are needed to understand the mechanistic basis of cholinergic and GABAergic modulation of repetition priming, the present data nevertheless show an important concurrence in behavioral impairments of repetition priming with regionally specific impairments in repetition related hemodynamic response reductions. These findings indicate that the neurochemical modulation of human learning and memory are now amenable to investigation in vivo.

\section{REFERENCES}

Backman L, Almkvist O, Nyberg L, Andersson J (2000) Functional changes in brain activity during priming in Alzheimer's disease. J Cogn Neurosci 12:134-141.

Badgaiyan RD, Schacter DL, Alpert NM (1999) Auditory priming within and across modalities: evidence from positron emission tomography. J Cogn Neurosci 11:337-348.

Blokland A (1996) Acetylcholine: a neurotransmitter for learning and memory? Brain Res Rev 21:285-300.

Bond A, Lader M (1974) The use of analogue scales in rating subjective feelings. Br J Med Psychol 47:211-218.

Brocher S, Artola A, Singer W (1992) Agonists of cholinergic and noradrenergic receptors facilitate synergistically the induction of long-term potentiation in slices of rat visual cortex. Brain Res 573:27-36.

Buckner RL, Goodman J, Burock M, Rotte M, Koutstaal W, Schacter D, Rosen B, Dale AM (1998) Functional-anatomic correlates of object priming in humans revealed by rapid presentation event-related fMRI. Neuron 20:285-296.

Buckner RL, Koutstaal W, Schacter DL, Rosen BR (2000) Functional MRI evidence for a role of frontal and inferior temporal cortex in amodal components of priming. Brain 123:620-640.

Buffett-Jerrott SE, Stewart SH, Teehan MD (1998) A further examination of the time-dependent effects of oxazepam and lorazepam on implicit and explicit memory. Psychopharmacology 138:344-353.

Caine ED, Weingartner H, Ludlow CL, Cudahy EA, Wehry S (1981) Qualitative analysis of scopolamine-induced amnesia. Psychopharmacology 74:74-80.

Curran HV, Pooviboonsuk P, Dalton JA, Lader MH (1991a) Differentiating the effects of centrally acting drugs on arousal and memory: an event-related potential study of scopolamine, lorazepam and diphenhydramine. Psychopharmacology 135:27-36.

Curran HV, Schifano F, Lader MH (1991b) Models for memory dysfunction? A comparison of the effects of scopolamine and lorazepam on memory, psychomotor performance and mood. Psychopharmacology 103:83-90.

Danion JM, Zimmermann MA, Willard-Schroeder D, Grange D, Welsch M, Imbs JL, Singer L (1990) Effects of scopolamine, trimipramine and diazepam on explicit memory and repetition priming in healthy volunteers. Psychopharmacology 102:422-424.

Demb JB, Desmond JE, Wagner AD, Vaidya CJ, Glover GH, Gabrieli JD (1995) Semantic encoding and retrieval in the left inferior prefrontal cortex: a functional MRI study of task difficulty and process specificity. J Neurosci 15:5870-5878.

Desimone R (1996) Neural mechanisms for visual memory and their role in attention. Proc Natl Acad Sci USA 93:13494-13499.

Ebert U, Siepmann M, Oertel R, Wesnes KA, Kirch W (1998) Pharmacokinetics and pharmacodynamics of scopolamine after subcutaneous administration. J Clin Pharmacol 38:720-726.

Frey KA, Koeppe RA, Mulholland GK, Jewett D, Hichwa R, Ehren- 
kaufer RL, Carey JE, Wieland DM, Kuhl DE, Agranoff BW (1992) In vivo muscarinic cholinergic receptor imaging in human brain with [11C]scopolamine and positron emission tomography. J Cereb Blood Flow Metab 12:147-154.

Friston KJ, Holmes AP, Worsley KP, Poline J-B, Frith CD, Frackowiak RSJ (1995) Statistical parametric maps in functional imaging: a general linear approach. Hum Brain Mapp 2:189-210.

Frith CD, Richardson JT, Samuel M, Crow TJ, McKenna PJ (1984) The effects of intravenous diazepam and hyoscine upon human memory. Q J Exp Psychol [A] 36:133-144.

Furey ML, Pietrini P, Haxby JV (2000) Cholinergic enhancement and increased selectivity of perceptual processing during working memory. Science 290:2315-2319.

George N, Dolan RJ, Fink GR, Baylis GC, Russell C, Driver J (1999) Contrast polarity and face recognition in the human fusiform gyrus. Nat Neurosci 2:574-580.

Giersch A, Boucart M, Danion JM, Vidailhet P, Legrand F (1995) Effects of lorazepam on perceptual integration of visual forms in healthy volunteers. Psychopharmacology 119:105-114.

Graf P, Mandler G (1984) Activation makes words more accessible, but not necessarily more retrievable. J Verb Learn Verb Behav 23:553-568.

Greenblatt DJ (1981) Clinical pharmacokinetics of oxazepam and lorazepam. Clin Pharmacokinet 6:89-105.

Henson R, Shallice T, Dolan R (2000) Neuroimaging evidence for dissociable forms of repetition priming. Science 287:1269-1272.

Josephs O, Henson RN (1999) Event-related functional magnetic resonance imaging: modelling, inference and optimization. Philos Trans R Soc Lond B Biol Sci 354:1215-1228.

Knopman D (1991). Unaware learning versus preserved learning in pharmacologic amnesia: similarities and differences. J Exp Psychol Learn Mem Cogn 17:1017-1029.

Legrand F, Vidailhet P, Danion JM, Grange D, Giersch A, van der Linden M, Imbs JL (1995) Time course of the effects of diazepam and lorazepam on perceptual priming and explicit memory. Psychopharmacology 118:475-479.

Li L, Miller EK, Desimone R (1993) The representation of stimulus familiarity in anterior inferior temporal cortex. J Neurophysiol 69:1918-1929.

Martinez R, Molchan SE, Lawlor BA, Thompson K, Martinson H, Latham G, Weingartner H, Sunderland T (1997) Minimal effects of dextroamphetamine on scopolamine-induced cognitive impairments in humans. Biol Psychiatry 41:50-57.

Miller EK, Desimone R (1993) Scopolamine affects short-term memory but not inferior temporal neurons. NeuroReport 4:81-84.

Nissen MJ, Knopman DS, Schacter DL (1987) Neurochemical dissociation of memory systems. Neurology 37:789-794.

Nuotto E (1983) Psychomotor, physiological and cognitive effects of scopolamine and ephedrine in healthy man. Eur J Clin Pharmacol 24:603-609.

Putcha L, Cintron NM, Tsui J, Vanderploeg JM, Kramer WG (1989) Pharmacokinetics and oral bioavailability of scopolamine in normal subjects. Pharmacol Res 6:481-485.

Rasmusson DD (2000) The role of acetylcholine in cortical synaptic plasticity. Behav Brain Res 115:205-218.

Richardson-Klavehn A, Gardiner JM (1998) Depth-of-processing ef- fects on priming in stem completion: tests of the voluntarycontamination, conceptual-processing, and lexical-processing hypotheses. J Exp Psychol Learn Mem Cogn 24:593-609.

Rusted JM, Warburton DM (1988) The effects of scopolamine on working memory in healthy young volunteers. Psychopharmacology 96:145-152.

Safer DJ, Allen RP (1971) The central effects of scopolamine in man. Biol Psychiatry 3:347-355.

Sannita WG, Fioretto M, Maggi L, Rosadini G (1987) Effects of scopolamine parenteral administration on the electroretinogram, visual evoked potentials, and quantitative electroencephalogram of healthy volunteers. Doc Ophthalmol 67:379-388.

Schacter DL, Buckner RL (1998) Priming and the brain. Neuron 20:185-195.

Schifano F, Curran HV (1994) Pharmacological models of memory dysfunction? A comparison of the effects of scopolamine and lorazepam on word valence ratings, priming and recall. Psychopharmacology $115: 430-434$

Squire LR, Ojemann JG, Miezin FM, Petersen SE, Videen TO, Raichle ME (1992) Activation of the hippocampus in normal humans: a functional anatomical study of memory. Proc Natl Acad Sci USA 89:1837-1841.

Sunderland T, Tariot PN, Cohen RM, Weingartner H, Mueller III EA, Murphy DL (1987) Anticholinergic sensitivity in patients with dementia of the Alzheimer type and age-matched controls. A dose-response study. Arch Gen Psychiatry 44:418-426.

Thompson-Schill SL, D'Esposito M, Kan IP (1999) Effects of repetition and competition on activity in left prefrontal cortex during word generation. Neuron 23:513-522.

Tsukada H, Kakiuchi T, Ando I, Shizuno H, Nakanishi S, Ouchi Y (1997) Regulation of cerebral blood flow response to somatosensory stimulation through the cholinergic system: a positron emission tomography study in unanesthetized monkeys. Brain Res 749:10-17.

Vidailhet P, Danion JM, Kauffmann-Muller F, Grange D, Giersch A, van der Linden M, Imbs JL (1994) Lorazepam and diazepam effects on memory acquisition in priming tasks. Psychopharmacology 115:397-406.

Vidailhet P, Danion JM, Chemin C, Kazes M (1999) Lorazepam impairs both visual and auditory perceptual priming. Psychopharmacology 147:266-273.

Vitiello B, Martin A, Hill J, Mack C, Molchan S, Martinez R, Murphy DL, Sunderland T (1997) Cognitive and behavioral effects of cholinergic, dopaminergic, and serotonergic blockade in humans. Neuropsychopharmacology 16:15-24.

Wagemans J, Notebaert W, Boucart M (1998) Lorazepam but not diazepam impairs identification of pictures on the basis of specific contour fragments. Psychopharmacology 138:326-333.

Wagner AD, Koutstaal W, Maril A, Schacter DL, Buckner RL (2000) Task-specific repetition priming in left inferior prefrontal cortex. Cereb Cortex 10:1176-1184.

Weinberger NM (1998) Physiological memory in primary auditory cortex: characteristics and mechanisms. Neurobiol Learn Mem 70:226-251.

Wiggs CL, Martin A (1998) Properties and mechanisms of perceptual priming. Curr Opin Neurobiol 8:227-233. 